\title{
Article \\ Elevated M-MDSCs in Circulation Are Indicative of Poor Prognosis in Diffuse Large B-Cell Lymphoma Patients
}

\author{
Zhitao Wang ${ }^{1}{ }^{\circledR}$, Rui Jiang ${ }^{2}$, Qian Li $^{1}$, Huiping Wang ${ }^{1}$, Qianshan Tao ${ }^{1}$ and Zhimin Zhai ${ }^{1, *}$ \\ 1 Department of Hematology, The Second Affiliated Hospital of Anhui Medical University, \\ Hefei 230601, China; wangzhitao@stu.ahmu.edu.cn (Z.W.); aydxyk101@163.com (Q.L.); \\ ahfymb1989@163.com (H.W.); ahbengbuwyl@163.com (Q.T.) \\ 2 Department of Hematology, The 901st Hospital of the Joint Logistics Support Force of PLA, \\ Hefei 230032, China; jianglang2002@sina.com \\ * Correspondence: aydxyk@163.com
}

Citation: Wang, Z.; Jiang, R.; Li, Q.; Wang, H.; Tao, Q.; Zhai, Z. Elevated M-MDSCs in Circulation Are Indicative of Poor Prognosis in Diffuse Large B-Cell Lymphoma Patients. J. Clin. Med. 2021, 10, 1768. https://doi.org/10.3390/jcm10081768

Academic Editors: Tadeusz Robak and Helen A. Papadaki

Received: 8 February 2021

Accepted: 9 April 2021

Published: 19 April 2021

Publisher's Note: MDPI stays neutral with regard to jurisdictional claims in published maps and institutional affiliations.

Copyright: (c) 2021 by the authors. Licensee MDPI, Basel, Switzerland. This article is an open access article distributed under the terms and conditions of the Creative Commons Attribution (CC BY) license (https:// creativecommons.org/licenses/by/ $4.0 /)$.

\begin{abstract}
Myeloid-derived suppressor cells (MDSCs) are defined as negative regulators that suppress the immune response through a variety of mechanisms, which usually cluster in cancer, inflammation, and autoimmune diseases. This study aims to investigate the correlation between M-MDSCs and the clinical features of diffuse large B-cell lymphoma (DLBCL) patients, as well as the possible accumulation mechanism of M-MDSCs. The level of M-MDSCs is significantly increased in newly diagnosed and relapsed DLBCL patients. Regarding newly diagnosed DLBCL patients, the frequency of M-MDSCs is positively correlated with tumor progression and negatively correlated with overall survival (OS). More importantly, the level of M-MDSCs can be defined as a biomarker for a poor prognosis in DLBCL patients. Additionally, interleukin-35 (IL-35) mediates the accumulation of M-MDSCs in DLBCL patients. Anti-IL-35 treatment significantly reduces levels of M-MDSCs in Ly8 tumor-bearing mice. Thus, M-MDSCs are involved in the pathological process of DLBCL. Targeting M-MDSCs may be a promising therapeutic strategy for the treatment of DLBCL patients.
\end{abstract}

Keywords: myeloid-derived suppressor cells; diffuse large B-cell lymphoma; interleukin-35; tumor progression; prognosis; immunosuppression

\section{Introduction}

Diffuse Large B-cell lymphoma (DLBCL) is the most common subtype of non-Hodgkin lymphoma, which represents about $30-40 \%$ of lymphomas [1]. The chemotherapy regimen of CHOP, defined as standard cyclophosphamide, doxorubicin, vincristine, and prednisolone or R-CHOP in combination with rituximab (R), has significantly improved survival outcomes in DLBCL patients [2]. However, approximately $30 \%$ of DLBCL patients in stage III and IV remain intractable, and the disease could eventually relapse [3]. During recent decades, the most commonly used standard indicator to assess prognosis in DLBCL is the international prognostic index (IPI), which is based on clinical parameters, including age, Eastern Cooperative Oncology Group(ECOG)performance status, Lactate Dehydrogenase (LDH)level, number of extranodal sites, and Ann Arbor stage. However, final survival is different in patients with identical IPI scores [4]. This means the IPI score system needs further improvement. It is notable that the IPI score system does not include the host tumor microenvironment (TME). The clinical outcome is dependent on many factors, including tumor histologic aggressiveness, immunologic status, and the tumor microenvironment, especially the immunosuppressive regulators [5].

MDSCs is a heterogeneous, immature immunosuppressive cell population which plays an important role in the occurrence and progression of tumors [6,7]. Considering mice, MDSC s can be divided into two types according to the expression of Gr-1, including the subtypes of Ly6G and Ly6C, which can be described as CD11b Ly $^{6} \mathrm{C}^{\text {low }} \mathrm{Ly}_{6 \mathrm{G}}{ }^{+}$ PMN-MDSCs and CD11b ${ }^{+}$Ly6C ${ }^{\text {high }} \mathrm{Ly}^{-} \mathrm{G}^{-}$M-MDSCs [8]. No human equivalent of Gr-1 
exists, so there is no uniform immunophenotype of human MDSCs. MDSCs promote tumor progression by inhibiting host immune responses [9]. MDSCs exert their immunosuppressive effect through different mechanisms, including direct contact and secreting immunomodulatory factors $[10,11]$. MDSCs have been described in hematological malignancies, including lymphoma [12], leukemia [13] and multiple myeloma [14]. However, the clinical significance of MDSCs in DLBCL has not been well investigated, especially combined with clinical parameters and prognoses.

It has been proven that MDSCs are found to accumulate in the presence of several biologic factors, such as cytokines, tumor cells, and complement proteins [15]. MDSCs can be generated by interleukin-6 (IL-6) and Granulocyte-macrophage Colony Stimulating Factor (GM-CSF)in vitro [16]. Interleukin-35 (IL-35), composed of an IL-27 subunit EBI3 and an IL-12 subunit p35, is an immunosuppressive cytokine defined as a negative regulator of T cell response $[17,18]$. Previously, it was reported that IL-35 could induce MDSC accumulation in the tumor microenvironment and promote tumor angiogenesis [19]. However, the role of IL-35 in the pathogenesis of DLBCL, as well as the effect on MDSCs accumulation, have not been well studied.

Here, we investigate the level and possible accumulation mechanism of M-MDSCs, which may be defined as a prognostic biomarker in DLBCL patients.

\section{Materials and Methods}

\subsection{Patient and Sample}

During January 2014 to June 2020, peripheral blood was collected from 65 newly diagnosed, 12 relapsed, 26 remission DLBCL patients, and 30 healthy donors when the state of the disease was defined, as shown in Table 1. All newly diagnosed DLBCL were classified according to World Health Organization standards [20]. Eighteen newly diagnosed DLBCL patients, who received at least 4 cycles of CHOP or R-CHOP regimen and had a complete response, were successfully followed up. Blood samples were obtained before receiving the 5 th regimen. The response was assessed according to the RECIL-2017 for lymphoma [21]. Patients with other medical conditions, including infectious diseases, autoimmune diseases, and other types of tumors, were excluded from the study. According to the guidelines of the Helsinki Declaration, the Ethics' Department of our University committee approved the research program. All patients and volunteers gave written informed consent.

Table 1. Characteristics of healthy donors and DLBCL patients.

\begin{tabular}{lccc}
\hline State of Disease at Sample Draw & No. of Patients & Average Age (Range) & Gender(M/F) \\
\hline $\begin{array}{l}\text { Newly diagnosed } \\
\text { Disease stage }\end{array}$ & 65 & $58.7(28-80)$ & $40 / 25$ \\
$\quad$ I-II & 37 & $57.8(30-80)$ & $20 / 17$ \\
III-IV & 28 & $59.1(28-78)$ & $20 / 8$ \\
B symptoms & & & \\
YES & 17 & $52.3(28-69)$ & $9 / 8$ \\
NO & 48 & $61.4(32-80)$ & $31 / 17$ \\
GCB & & & \\
YES & 38 & $58.9(28-76)$ & $23 / 15$ \\
NO & 27 & $58.5(30-80)$ & $17 / 10$ \\
LDH & & & \\
Normal & 30 & $60.4(30-80)$ & $20 / 10$ \\
Increased & 35 & $57.3(28-78)$ & $20 / 15$ \\
IPI score & & & \\
0-2 & 41 & $55.8(28-76)$ & $24 / 17$ \\
3-5 & 24 & $63.7(32-80)$ & $16 / 8$ \\
Relapsed & 12 & $58.3(36-62)$ & $9 / 3$ \\
Remission & 26 & $62.3(31-76)$ & $14 / 12$ \\
Healthy donors & 30 & $60.1(30-78)$ & $18 / 12$ \\
\hline
\end{tabular}

GCB germinal center B-cell-like, LDH lactate dehydrogenase, IPI International Prognostic Index. B symptoms: B symptoms refer to systemic symptoms of fever, night sweats, and weight loss which can be associated with diffuse Large B-cell lymphoma (DLBCL). 


\subsection{Flow Cytometry (FCM) Analysis}

Monoclonal antibodies (mAbs) were purchased from Beckman Coulter-Immunotech: FITC-labeled anti-CD14 (clone No. 116), APC-labeled anti-CD14 (clone No. RMO52), PE-labeled anti-HLA-DR (clone No. B8.12.2), ECD-labeled anti-HLA-DR (clone No. Immu375), PE-labeled anti-CD4 (clone No. 13B8.2); Mouse FITC-labeled anti-Ly6G (clone No. RB6-8C5), Mouse APC-labeled anti-Ly6C (clone No. ab93550), and Mouse PE-labeled anti-CD11b (clone No. M1/70).

Peripheral blood mononuclear cells (PBMCs) were layered over Ficoll-Hypaque (Amersham Biosciences, Sweden) and centrifuged at $500 \times g$ for $25 \mathrm{~min}$. Following density gradient centrifugation, PBMCs and plasma were collected. One hundred microliters of PBMCs were used for flow cytometry (Flow Cytometer: FC500 MPL, Beckman Coulter, Brea, CA, USA) and the rest for real-time PCR analysis. Ten thousand cells were analyzed by FCM for each sample. Isotype-matched antibodies were used as controls. During this study, the immunophenotype of M-MDSCs was defined as CD14 ${ }^{+} \mathrm{HLA}-\mathrm{DR}{ }^{-/ \mathrm{low}}$ in

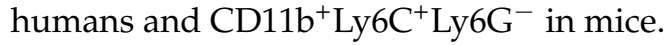

\subsection{Cytokine Assay}

A human IL-35 enzyme linked immunosorbent assay (ELISA) kit (Biolegend, San Diego, CA, USA) was used to measure the concentration of IL-35 according to the instructions. Each sample was run in duplicate.

\subsection{RT-PCR Analysis}

TRizol reagent (Invitrogen, Carlsbad, CA, USA) was used to extract total RNA from PBMCs. First-strand cDNA was synthesized using the First-Strand Synthesis System (TaKaRa, Dalian, China). SYBR Green PCR Master mix (TaKaRa, Dalian, China) was used to perform the Real-time PCR.

An Applied Biosystems 7500 Real-time Polymerase Chain Reaction (RT-PCR) system was used to analyze the subunit EBI3 and p35 of IL-35. The following primers were employed in each reaction: EBI3, forward $5^{\prime}$-GGCAAGTAGCAAG GGCTTC-3' and reverse 5'-AGTCGGTCATCTGAGGTTGC-3'; p35, forward 5'-TCCTCCTTGAAGAACCGGA-3' and reverse $5^{\prime}$-TGA CAACGGTTTGGAGGGAC-3'. Glyceraldehyde phosphate dehydrogenase (GAPDH) was used as the control: forward 5'-CAGGAGGCCATTGCTGATGAT- $3^{\prime}$ and reverse $5^{\prime}$-GAAGGCTGGGGCTCATTT- $3^{\prime}$. The thermal cycling conditions are described as follows: Following an initial denaturation step at $95^{\circ} \mathrm{C}$ for $30 \mathrm{~s}, 40$ cycles of profile were carried out: $95^{\circ} \mathrm{C}, 5 \mathrm{~s} ; 60^{\circ} \mathrm{C}, 34 \mathrm{~s}$. Relative transcripts were determined by the formular: $2^{-(\text {CTtarget-CTcontrol) }}$.

\subsection{Cell Culture and Cytokine Induction}

PBMCs $\left(1 \times 10^{6}\right)$ were isolated from 5 healthy controls and incubated in the presence or absence of rh-IL-35 (Recombinant Human Interleukin-35, $50 \mathrm{ng} / \mathrm{mL}$, Sino Biological Inc., Beijing, China) in a 24-well plate for $72 \mathrm{~h}$ in vitro. To enhance cell viability, rh-GM-CSF (10 ng/mL; Sigma, St. Louis, MI, USA) was added to the mixture [22]. PBMCs were cultured alone as a control. Three replicates were performed for each condition. The cells were cultured at $37^{\circ} \mathrm{C}$ with an RPMI- 1640 medium in a humidified $\mathrm{CO}_{2}$-containing atmosphere.

\subsection{Assay for Autologous T-Cell Proliferation}

An MoFlo XDP cell sorter (Beckman Coulter, USA) was used to isolate CD14 ${ }^{+} \mathrm{HLA}-$ $\mathrm{DR}^{-/ \text {low }}{ }^{-}$DSCs(M-MDSCs) and CD14 ${ }^{+} \mathrm{HLA}-\mathrm{DR}^{+}$cells. Autologous $\mathrm{CD}^{+} \mathrm{T}$ cells were sorted by anti-CD4 beads (Miltenyi Biotec, Bergisch Gladbach, Germany) from the same healthy control. The purity of sorted cells was $>95 \%$.

$\mathrm{CD}^{+} \mathrm{T}$ cells were incubated with CFSE $(0.5 \mu \mathrm{M}$, Invitrogen, USA). Next, M-MDSCs and $\mathrm{CD} 14^{+} \mathrm{HLA}^{-D R^{+}}$cells were cocultured with CFSE-labeled CD4 ${ }^{+} \mathrm{T}$ cells, respectively, in a 96-well plate at the ratio of 1:1. $\mathrm{CD} 4^{+} \mathrm{T}$ cells were cultured alone as a positive control. 
All cells were cultured in an RPMI-1640 medium with anti-CD3 $(2 \mu \mathrm{g} / \mathrm{mL})$, anti-CD28 $(5 \mu \mathrm{g} / \mathrm{mL})$. The suppressive ability of M-MDSCs on $\mathrm{CD} 4^{+} \mathrm{T}$ cells was analyzed 3 days later. Supernatants of culture were obtained and stored at $-80{ }^{\circ} \mathrm{C}$ until used. Interferon- $\gamma$ (IFN- $\gamma$ ) was detected using an ELISA Kit (R\&D System; ESM, Minneapolis, MN, USA).

\subsection{Animal Models and Treatments}

To establish the Ly8 DLBCL tumor mouse model, $1 \times 10^{6}$ Ly8 cells were subcutaneously injected into the flank of NOD-SCID mice to form tumors. Seven days after tumor cell injection (when the tumor surface area was $\sim 100 \mathrm{~mm}^{2}$ ), mice received weekly administration of anti-IL-35 (Clone V1.4F5.25) or $\mathrm{IgG}_{2 \mathrm{~b}}$ antibody $(100 \mu \mathrm{g})$ where indicated. Additional NOD-SCID mice without any treatment were regarded as the control group. According to the experimental scheme, the mice were killed on Day 23 after tumor inoculation, and blood was collected. Blood was subsequently prepared for flow cytometry analysis of the M-MDSCs population.

\subsection{Statistical Analysis}

Statistical analysis was conducted with the SPSS 17.0 software (SPSS Inc, Chicago, IL, USA). The Mann-Whitney U-test, Student's t test, and one-way ANOVA were used to determine the statistical significance when appropriate. To evaluate the correlations, Spearman's coefficient test was used. The overall survival rate (OS) was defined as the time from the start of treatment to death from any cause. The OS was determined using the Kaplan-Meier method. The difference was assessed using the log-rank test. A COX proportional hazard regression analysis identified independent prognostic factors for OS. Regarding all analyses, a $p$ value $<0.05$ was considered significant.

\section{Results}

\subsection{Increased M-MDSCs in Newly Diagnosed and Relapsed DLBCL Patients}

Cells with the immunophenotype of $\mathrm{CD} 14^{+} \mathrm{HLA}-\mathrm{DR}{ }^{-/ \text {low }}$ were defined as monocytic MDSCs (M-MDSCs). Compared to the 30 healthy controls, a significantly increased frequency of M-MDSCs was found in the 65 newly diagnosed DLBCL patients $(4.7 \pm 3.6 \%$ vs. $25.4 \pm 12.3 \%, p<0.01$, Figure 1A,B). However, no significant difference existed between the groups by age, gender, and GCB, or B symptoms (Figure 1C,D).

A

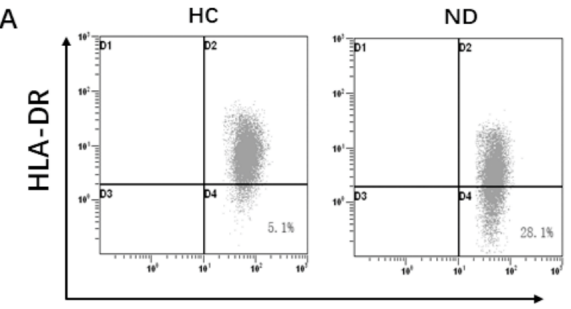

CD14
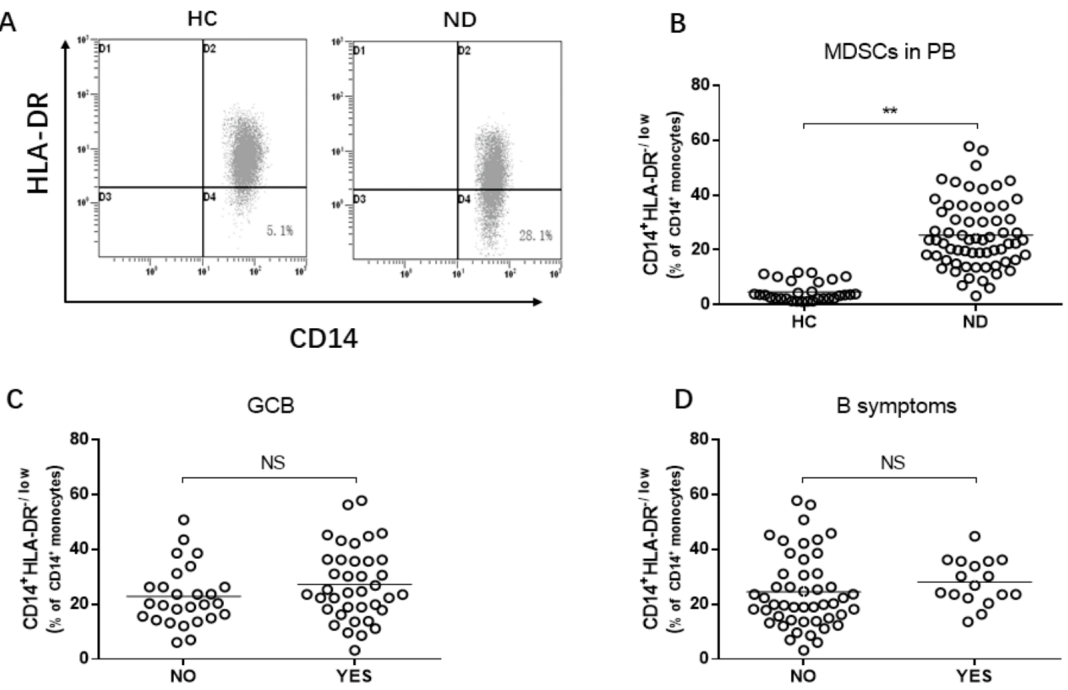

Figure 1. The level of M-MDSCs in DLBCL patients. (A) FCM dot plots demonstrate the frequency of M-MDSCs. (B) M-MDSCs in DLBCL patients compared to healthy controls. (C) M-MDSCs in GCB and no-GCB DLBCL patients. (D) M-MDSCs in A and B symptoms in DLBCL patients. HC, healthy control; ND, newly diagnosed. ${ }^{* *} p<0.01$. 


\subsection{M-MDSCs Levels Correlate with Disease Progression in Newly Diagnosed DLBCL Patients}

Many clinicopathological factors were used to indicate disease progression, including the Ann Arbor Stage, LDH level, and disease status. DLBCL patients with stage III-IV were found to have higher levels of M-MDSCs compared to patients with early stage I-II (32.4 \pm $12.2 \%$ vs. $20.1 \pm 9.5 \%, p<0.01$, Figure $2 \mathrm{~A}$ ). Higher levels of M-MDSCs were observed in DLBCL patients with increased LDH levels $(30.6 \pm 11.3 \%)$ compared with the LDH normal group (19.4 $\pm 10.7 \%, p<0.01$, Figure 2B). An increased frequency of M-MDSCs existed in newly diagnosed $(25.4 \pm 12.3 \%)$ and relapsed $(34.2 \pm 17.0 \%)$ DLBCL patients compared to remission (10.1 $\pm 3.5 \%$, Figure $2 \mathrm{C}$ ) patients. Regarding the 18 patients who received four cycles of CHOP or R-CHOP regimens of chemotherapy, the frequency of M-MDSCs was significantly decreased after therapy $(28.6 \pm 8.5 \%$ vs. $13.2 \pm 5.3 ; p<0.01$, Figure 2D).

A
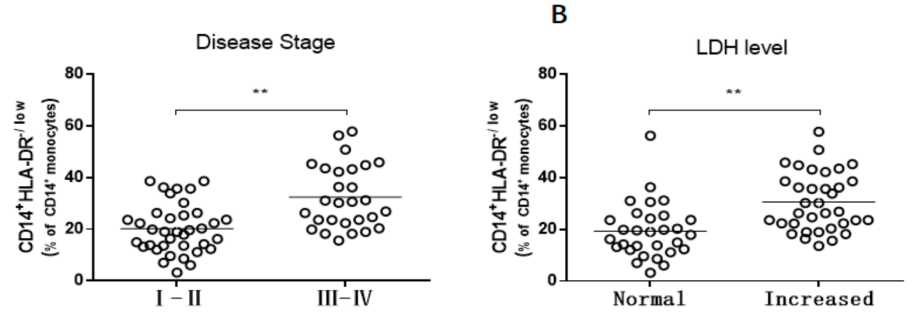

C

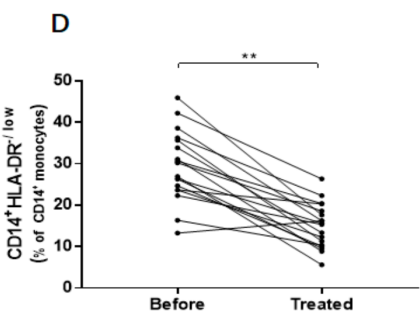

Figure 2. M-MDSCs were associated with tumor progression in DLBCL patients. (A) M-MDSCs were associated with DLBCL clinical stages. (B) High LDH level DLBCL patients had an increased frequency of M-MDSCs. (C) Newly diagnosed and relapsed patients had higher levels of M-MDSCs than remission patients. (D) The levels of M-MDSCs significantly decreased after therapy. ND, newly diagnosed; Rel, relapsed; Rem, remission. ${ }^{*} p<0.05 ;{ }^{* *} p<0.01$.

\subsection{The Association Between M-MDSCs and Prognosis of DLBCL Patient}

First, the relationship between the IPI score and the expansion of M-MDSCs was investigated. We found increased levels of M-MDSCs in the high IPI score (IPI score: $3-5$ ) DLBCL patients compared to the low IPI score (IPI score: $0-2)$ patients $(35.3 \pm 11.4 \%$ vs. $19.6 \pm 8.7 ; p<0.01$, Figure $3 \mathrm{~A}$ ). Furthermore, the IPI score was significantly and positively associated with the frequency of M-MDSCs $(r=0.65, p<0.01$, Figure 3B).

The follow-up time was 5-76 months from January 2014 to June 2020. The negative correlation between the OS and the frequency of M-MDSCs was further validated $(\mathrm{r}=0.47$, $p<0.01$, Figure 3C). Based on the median value frequency of M-MDSCs $(25.4 \%)$, DLBCL patients were divided into two groups. Concerning the low group $(n=39)$, M-MDSCs levels were defined as less than or equal to $25.4 \%$. Regarding the high group $(n=26)$, M-MDSCs levels were greater than $25.4 \%$. A Kaplan-Meier analysis showed the OS of DLBCL patients with low M-MDSC levels was significantly longer than those with high M-MDSC levels ( $p<0.01$, Figure 3D).

A univariate analysis of prognostic factors for OS, including age, gender, disease stage, LDH level, B symptoms, GCB, IPI score, and the frequency of M-MDSCs, was conducted. Logically, disease stage, IPI score, LDH level, and the frequency of M-MDSCs were significant prognostic indicators for OS $(p<0.05$; Table 2). When adjusted for the key clinical prognostic factors, a multivariate Cox regression analysis also was performed. The 
results showed the IPI score and M-MDSC levels were associated with prognosis and could be recognized as independent prognostic factors for DLBCL patients $(p<0.05$, Table 2$)$.

A

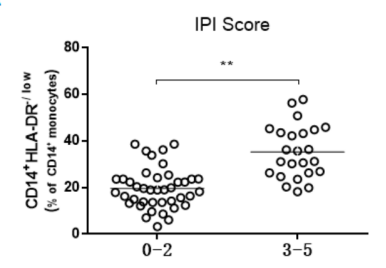

c

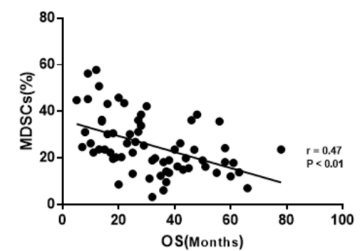

$B$

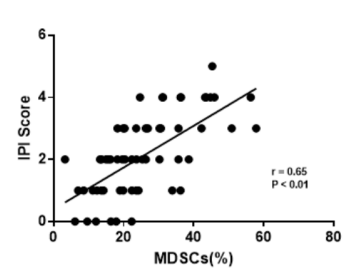

D

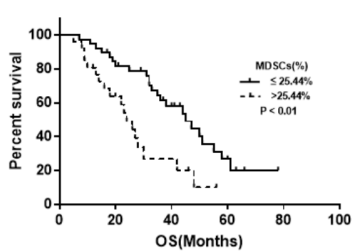

Figure 3. Circulating M-MDSCs was a prognostic factor in DLBCL patients. (A) High IPI score DLBCL patients had an increased frequency of M-MDSCs. (B) M-MDSCs levels were positively associated with the IPI score. (C) M-MDSC levels were negatively correlated with the OS. (D) Short OS were shown in high M-MDSCs groups. The cut-off value is the median of M-MDSC levels. ${ }^{* *} p<0.01$.

Table 2. Univariate and Multivariate analysis of prognostic factors in patients with DLBCL.

\begin{tabular}{|c|c|c|c|c|c|c|}
\hline \multirow{2}{*}{ Factor } & \multicolumn{3}{|c|}{$\begin{array}{l}\text { Univariate } \\
\text { Analysis }\end{array}$} & \multicolumn{3}{|c|}{$\begin{array}{c}\text { Multivariate } \\
\text { Analysis }\end{array}$} \\
\hline & HR & $95 \% \mathrm{CI}$ & $p$ & HR & $95 \%$ CI & $p$ \\
\hline $\begin{array}{c}\text { Age (year) } \\
(\leq 60 \text { vs. }>60) \\
\text { Gender }\end{array}$ & 1.511 & $0.811-2.817$ & 0.194 & & & \\
\hline (Male vs. Female) & 1.127 & $0.601-2.112$ & 0.709 & & & \\
\hline $\begin{array}{l}\text { Disease stage } \\
\text { (I-II vs. III-IV) }\end{array}$ & 3.026 & $1.591-5.755$ & 0.001 & 2.184 & $0.916-5.205$ & 0.078 \\
\hline $\begin{array}{c}\text { B symptoms } \\
\text { (No vs. Yes) } \\
\text { GCB }\end{array}$ & 1.339 & $0.710-2.523$ & 0.367 & & & \\
\hline $\begin{array}{c}\text { (No vs. Yes) } \\
\text { LDH }\end{array}$ & 1.060 & $0.576-1.981$ & 0.855 & & & \\
\hline $\begin{array}{c}\text { (Normal vs. Increased) } \\
\text { IPI score }\end{array}$ & 0.348 & $0.136-0.887$ & 0.027 & 1.461 & $0.529-4.035$ & 0.464 \\
\hline $\begin{array}{c}(0-2 \text { vs. } 3-5) \\
\text { MDSCs }\end{array}$ & 2.976 & $1.549-5.717$ & 0.001 & 0.271 & $0.103-0.712$ & 0.008 \\
\hline$(\leq 25.44 \%$ vs. $>25.44 \%)$ & 2.707 & $1.409-5.199$ & 0.003 & 2.682 & $1.198-6.004$ & 0.016 \\
\hline
\end{tabular}

OS overall survival, HR hazard ratio, CI confidence interval, GCB germinal center B-cell-like, LDH lactate dehydrogenase, IPI International Prognostic Index. MDSC level (high/low) is based on the median value of the MDSC frequency.

\subsection{Increased IL-35 Induce the M-MDSCs Expansion}

IL-35, as a novel inhibitory cytokine, is composed of the subsets of p35 and EBI3 [23]. First, relative expressions of p35 and EBI3 mRNA were detected. Significantly increased expression of p35 mRNA was found in DLBCL patients compared to the healthy controls $(61.49 \pm 13.58$ vs. $20.34 \pm 7.06 ; p<0.01$, Figure 4 A), as well as expression of EBI3 mRNA $(21.88 \pm 6.06$ vs. $4.76 \pm 2.43 ; p<0.01$, Figure $4 \mathrm{~B})$. Next, the concentration of IL-35 was investigated. There was a significantly higher concentration of IL-35 in DLBCL patients compared to the healthy controls $(103.39 \pm 57.53 \mathrm{pg} / \mathrm{mL}$ vs. $65.18 \pm 18.23 \mathrm{pg} / \mathrm{mL} ; p<0.01$, Figure 4C). 


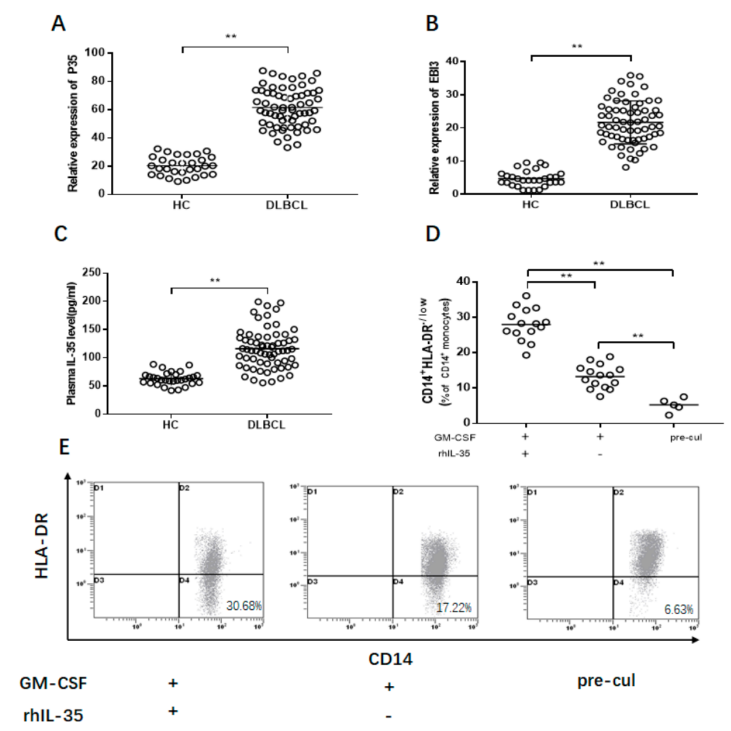

Figure 4. The levels of IL-35 in DLBCL patients and their effect on M-MDSCs. (A) p35 and (B) EBI3 mRNA expression in DLBCL patients and healthy controls. (C) The concentration of IL-35 in DLBCL patients and healthy controls. (D) IL-35 induced the expansion of M-MDSCs in vitro. (E) FACS dot plots show the proliferation of M-MDSCs induced with IL-35. Pre-cul, pre-culture; ${ }^{* *} p<0.01$.

Next, the effect of IL-35 on the accumulation of M-MDSCs was investigated. First, PBMCs, isolated from healthy controls, were cultured with rhIL-35(50 ng/mL) for $72 \mathrm{~h}$ in vitro. GM-CSF (10 ng/mL; Sigma) was used to support cell viability. The percentage of M-MDSCs was significantly increased with stimulation of IL-35 compared to the control $(p<0.01$, Figure 4D,E).

\subsection{IL-35-Induced M-MDSCs Suppress CD4 $4^{+}$T Cell Response}

Compared with the CD14 ${ }^{+} \mathrm{HLA}_{-\mathrm{DR}}^{+}$cells, M-MDSC s had a strong ability to suppress $\mathrm{CD}^{+} \mathrm{T}$ cell proliferation and reduce the production of IFN- $\gamma(p<0.01$, Figure 5A,B). CD14 ${ }^{+} \mathrm{HLA}_{-} \mathrm{DR}^{+}$cells did not have this capacity $(p<0.01$, Figure $5 \mathrm{C})$.
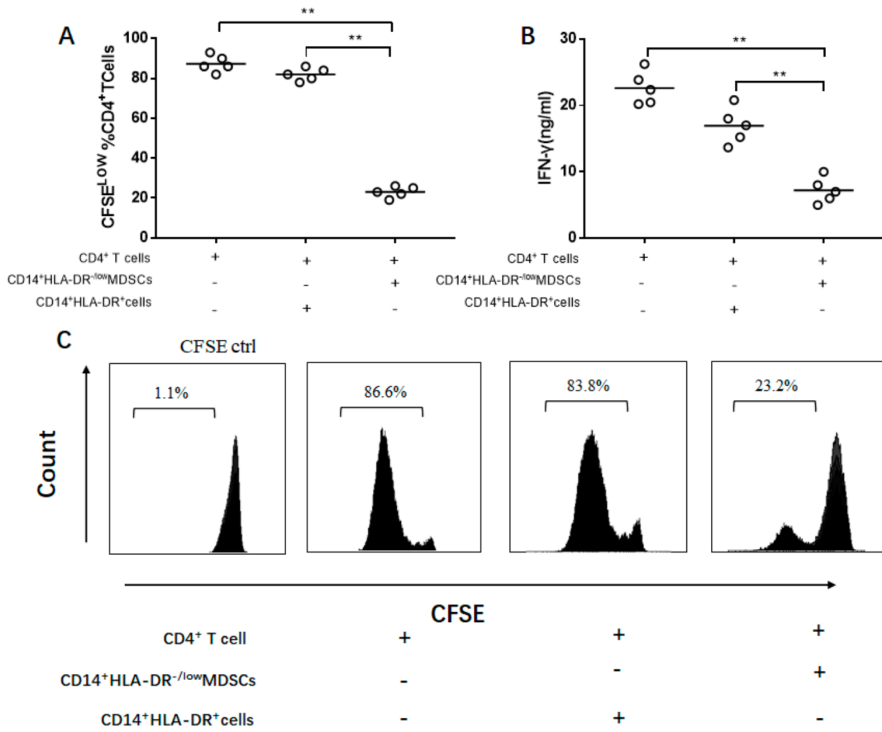

Figure 5. Functional analysis of M-MDSCs on $C D 4^{+} \mathrm{T}$ cells. (A) CFSE-labeled CD4 ${ }^{+} \mathrm{T}$ cells were suppressed by M-MDSCs, (B) associated with a reduction of IFN- $\gamma$. (C) A flow cytometry histogram shows the suppressive activity of M-MDSCs. Ctrl, control, ${ }^{* *} p<0.01$. 


\subsection{Anti-IL-35 Treatment Block M-MDSC Expansion In Vivo}

To study the effect of IL-35 on MDSC expansion, NOD-SCID mice injected with Ly8 DLBCL tumor cells were treated with anti-IL-35 or $\mathrm{IgG}_{2 \mathrm{~b}}$ antibodies (Figure 6A). Additional NOD-SCID mice without any treatment were regarded as the control group. Compared with the IgG2b antibody, Anti-IL-35 treatment significantly reduced the plasma levels of IL-35 in Ly8 tumor-bearing mice ( $p<0.01$, Figure $6 \mathrm{~B})$. Furthermore, obviously decreased levels of M-MDSCs were found in mice treated with Anti-IL-35 $(p<0.01$, Figure 6C,D, $\mathrm{P} 4$ region).
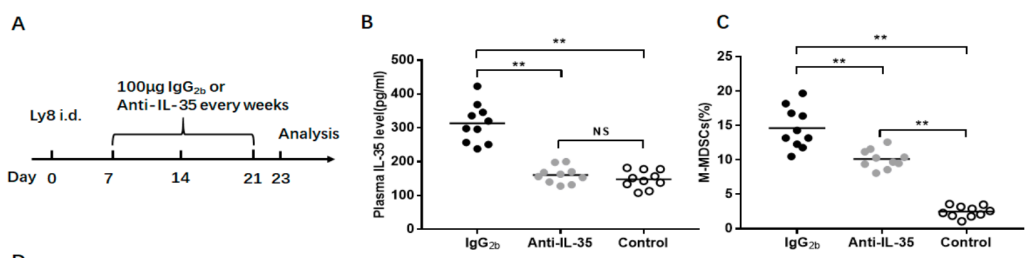

D

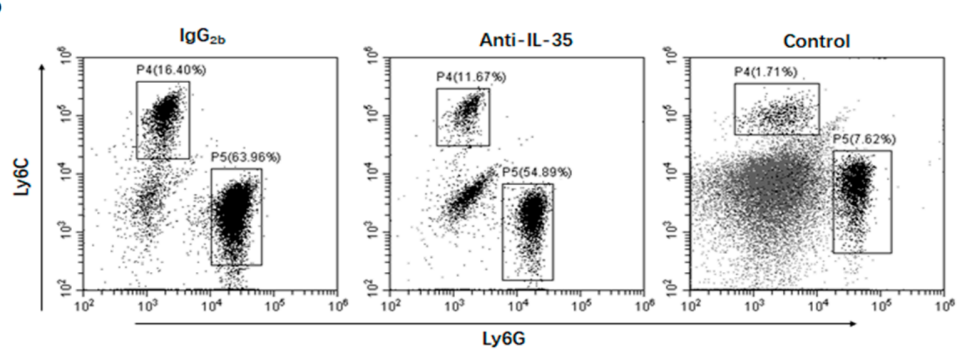

Figure 6. Effect of IL-35 on M-MDSC expansion in vivo. (A) Schematic representation of the experimental design. (B) Anti-IL-35 treatment reduced levels of IL-35. (C) Anti-IL-35 blocked the M-MDSC expansion in vivo. (D) Flow cytometry dot plots show the level of M-MDSCs in the three groups (P4 region). ${ }^{* *} p<0.01$.

\section{Discussion}

MDSCs are a heterogeneous, immature immunosuppressive cell population, which has a positive role in tumorigenesis and tumor progression [24,25]. It has been proven that the frequency of MDSCs in colorectal cancer [26], breast cancer [27], and multiple myeloma [14] is significantly increased and closely related to the progression of the tumor. The immunosuppressive capacity of M-MDSCs was more intensely compared with GMDSCs in the tumor microenvironment [28]. Additionally, G-MDSCs were absent from PBMCs, and were only detected in whole blood in several malignancy patients [29]. Thus, G-MDSCs are less studied than M-MDSCs in previous reports. M-MDSCs focused our attention during this study.

During this study, the clinical parameters and prognostic significance associated with the frequency of M-MDSCs was investigated in patients with DLBCL. First, the frequency of M-MDSCs was significantly increased in newly diagnosed and relapsed DLBCL patients and closely related to disease progression (disease stage, LDH levels, and IPI score). Following chemotherapy, a significant decrease in the levels of M-MDSCs was found. This indicated that the level of M-MDSCs reflected the disease progress of DLBCL patients. Accompanying the reduction of the tumor burden, inflammatory cytokines secreted by DLBCL cells significantly decreased, accompanied by reduced levels of MMDSCs. Concerning DLBCL patients, significant changes in M-MDSCs can be used to indicate the outcome of chemotherapy.

During a previous study [30,31], the levels of MDSCs were significantly different between GCB and non-GCB in poor and very good risk groups in DLBCL patients. Following chemotherapy, no significant difference existed in the five-year OS between GCB and non-GCB-DLBCL patients. The levels of M-MDSCs can be indicated as a biomarker to evaluate the prognosis of DLBCL patients. During the present study, the frequency of M-MDSCs was positively associated with the IPI score and negatively correlated with the OS in DLBCL patients. The group with a longer OS had a lower frequency of M-MDSCs. 
This proved that the level of M-MDSCs was a factor affecting the OS in DLBCL patients. The results from a Cox regression analysis showed that M-MDSCs were associated with a poor prognosis and could be defined as a prognostic biomarker for DLBCL patients.

Previous studies have shown that IL-35 exerts an immunosuppressive effect on inflammation related diseases [18]. The concentration of IL-35 increased in various tumor patients, including colorectal cancer [32], acute myeloid leukemia [17], and hepatocellular carcinoma [33]. However, to our best knowledge, there were few reports investigating IL-35 in DLBCL patients, as well as the effect of IL-35 on MDSCs. Wang et al. found that IL-35 has a strong ability to induce $\mathrm{CD} 11 \mathrm{~b}^{+} \mathrm{Gr} 1^{+}$myeloid cell accumulation in the mouse tumor microenvironment [19]. The influence of IL-35 on the accumulation of human M-MDSCs has not been reported before, to our knowledge. Data from the present study demonstrated that the concentrations of IL-35, as well as p35 and EBI3 mRNA expressions, were significantly elevated in DLBCL patients. Additionally, IL-35 has an ability to induce M-MDSC accumulation. Regarding the experiment in mice, the direct depletion of IL-35 using a neutralizing antibody contributes to the decrease in M-MDSC accumulations in mice with Ly8 DLBCL tumors. Interestingly, a decreased level of G-MDSCs also was found with the treatment of Anti-IL-35. These results collectively demonstrated that Anti-IL-35 treatment significantly blocked M-MDSC expansion. IL-35 exerted an immunosuppressive effect by inducing M-MDSC accumulations. Recently, however, the specific mechanism and molecular pathway of IL-35-induced M-MDSC expression remains to be further elucidated.

The uniform phenotypic marker for MDSCs is still lacking. The critical feature in the identification of MDSCs was the immunosuppressive capacity [34]. To identify whether the

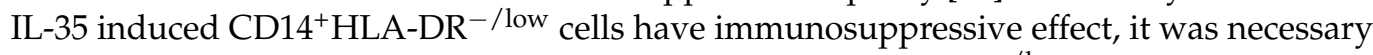
to evaluate the immunosuppressive activities of $\mathrm{CD} 14^{+} \mathrm{HLA}-\mathrm{DR}^{-/ \text {low }}$ cells on autologous $\mathrm{T}$ cell proliferation and IFN- $\gamma$ production. It was found that $\mathrm{CD} 14^{+} \mathrm{HLA}-\mathrm{DR}{ }^{-/ \text {low }}$ cells significantly suppressed the proliferation of $\mathrm{CD}^{+} \mathrm{T}$ cells compared to the CD14 ${ }^{+} \mathrm{HLA}-$ $\mathrm{DR}^{+}$cells. Moreover, the production of IFN- $\gamma$ was significantly decreased in $\mathrm{CD}^{+} \mathrm{T}$ cells co-cultured with $\mathrm{CD} 14^{+} \mathrm{HLA}-\mathrm{DR}{ }^{-/ \text {low }}$ cells. Strikingly, $\mathrm{CD} 14^{+} \mathrm{HLA}^{-\mathrm{DR}}{ }^{-/ \text {low }}$ cells exerted a strong suppressive ability on T cell proliferation and IFN- $\gamma$ production. Hence, $\mathrm{CD}_{1}{ }^{+} \mathrm{HLA}^{-\mathrm{DR}^{-/ \text {low }}}$ cells could be considered as M-MDSCs in DLBCL patients.

\section{Conclusions}

Increased levels of M-MDSCs are positively associated with tumor progression and inversely correlated with OS in DLBCL patients. The level of M-MDSCs is associated with prognosis and could be defined as a prognostic indicator for DLBCL patients. Additionally, IL-35 has an obvious ability to induce the accumulation of M-MDSCs. Therefore, M-MDSCs participate in immune escape and have a prognostic value in DLBCL patients. Targeting M-MDSCs may be a promising therapeutic strategy for DLBCL patients.

Author Contributions: Conceptualization, Z.Z.; resources, Z.W., R.J., and Q.L.; investigation, Z.W., and H.W.; resources, Z.W., R.J., Q.T., and Q.L.; statistical analysis, Z.W. and Q.L.; writing—original draft preparation, Z.W., Q.L., and H.W.; writing—review and editing, Z.W. and Z.Z.; supervision, $Z . Z$. All authors have read and agreed to the published version of the manuscript.

Funding: This work was supported by the Natural Science Foundation of China (81670179) and Anhui province (2008085QH372).

Institutional Review Board Statement: The study was approved by the local ethical committee of the Second Affiliated Hospital of Anhui Medical University (protocol code: 20140322; date of approval: 01.02.2014).

Informed Consent Statement: Informed consent was obtained from all subjects in volved in the study.

Data Availability Statement: The data presented in this study are available on request from the corresponding author.

Conflicts of Interest: All authors declare no conflict of interest. 


\section{References}

1. Armitage, J.O.; Gascoyne, R.D.; Lunning, M.A.; Cavalli, F. Non-Hodgkin lymphoma. Lancet 2017, 390, 298-310. [CrossRef]

2. Coiffier, B. Rituximab in combination with CHOP improves survival in elderly patients with aggressive non-Hodgkin's lymphoma. Semin. Oncol. 2002, 29, 18-22. [CrossRef] [PubMed]

3. Shanbhag, S.; Ambinder, R.F. Hodgkin lymphoma: A review and update on recent progress. CA A Cancer J. Clin. 2018, 68, 116-132. [CrossRef] [PubMed]

4. Tomita, N.; Yokoyama, M.; Yamamoto, W.; Watanabe, R.; Shimazu, Y.; Masaki, Y.; Tsunoda, S.; Hashimoto, C.; Murayama, K.; Yano, T.; et al. The standard international prognostic index for predicting the risk of CNS involvement in DLBCL without specific prophylaxis. Leuk. Lymphoma 2017, 59, 97-104. [CrossRef]

5. Chan, A.; Dogan, A. Prognostic and Predictive Biomarkers in Diffuse Large B-cell Lymphoma. Surg. Pathol. Clin. 2019, 12, 699-707. [CrossRef]

6. Pyzer, A.R.; Cole, L.; Rosenblatt, J.; Avigan, D.E. Myeloid-derived suppressor cells as effectors of immune suppression in cancer. Int. J. Cancer 2016, 139, 1915-1926. [CrossRef]

7. Chen, M.-F.; Tsai, M.-S.; Chen, W.-C.; Chen, P.-T. Predictive Value of the Pretreatment Neutrophil-to-Lymphocyte Ratio in Head and Neck Squamous Cell Carcinoma. J. Clin. Med. 2018, 7, 294. [CrossRef]

8. Chang, C.-J.; Yang, Y.-H.; Chiu, C.-J.; Lu, L.-C.; Liao, C.-C.; Liang, C.-W.; Hsu, C.-H.; Cheng, A.-L. Targeting tumor-infiltrating Ly6G+ myeloid cells improves sorafenib efficacy in mouse orthotopic hepatocellular carcinoma. Int. J. Cancer 2018, 142, 1878-1889. [CrossRef]

9. Marvel, D.; Gabrilovich, D.I. Myeloid-derived suppressor cells in the tumor microenvironment: Expect the unexpected. J. Clin. Investig. 2015, 125, 3356-3364. [CrossRef]

10. Talmadge, J.E.; Gabrilovich, D.I. History of myeloid-derived suppressor cells. Nat. Rev. Cancer 2013, 13, 739-752. [CrossRef]

11. Iwata, T.; Kondo, Y.; Kimura, O.; Morosawa, T.; Fujisaka, Y.; Umetsu, T.; Kogure, T.; Inoue, J.; Nakagome, Y.; Shimosegawa, T. PD-L1+MDSCs are increased in HCC patients and induced by soluble factor in the tumor microenvironment. Sci. Rep. 2016, 6, 39296. [CrossRef]

12. Zhang, H.; Li, Z.-L.; Ye, S.-B.; Ouyang, L.-Y.; Chen, Y.-S.; He, J.; Huang, H.-Q.; Zeng, Y.-X.; Zhang, X.-S.; Li, J. Myeloid-derived suppressor cells inhibit T cell proliferation in human extranodal NK/T cell lymphoma: A novel prognostic indicator. Cancer Immunol. Immunother. 2015, 64, 1587-1599. [CrossRef]

13. Yin, J.; Wang, C.; Huang, M.; Mao, X.; Zhou, J.; Zhang, Y. Circulating CD14(+) HLA-DR(-/low) myeloid-derived suppressor cells in leukemia patients with allogeneic hematopoietic stem cell transplantation: Novel clinical potential strategies for the prevention and cellular therapy of graft-versus-host disease. Cancer Med. 2016, 5, 1654-1669. [CrossRef]

14. Wang, Z.; Zhang, L.; Wang, H.; Xiong, S.; Li, Y.; Tao, Q.; Xiao, W.; Qin, H.; Wang, Y.; Zhai, Z. Tumor-induced CD14+HLADR-/low myeloid-derived suppressor cells correlate with tumor progression and outcome of therapy in multiple myeloma patients. Cancer Immunol. Immunother. 2015, 64, 389-399. [CrossRef]

15. Xiao, P.; Wan, X.; Cui, B.; Liu, Y.; Qiu, C.; Rong, J.; Zheng, M.; Song, Y.; Chen, L.; He, J.; et al. Interleukin 33 in tumor microenvironment is crucial for the accumulation and function of myeloid-derived suppressor cells. OncoImmunology 2015, 5, e1063772. [CrossRef]

16. Lechner, M.G.; Liebertz, D.J.; Epstein, A.L. Characterization of Cytokine-Induced Myeloid-Derived Suppressor Cells from Normal Human Peripheral Blood Mononuclear Cells. J. Immunol. 2010, 185, 2273-2284. [CrossRef]

17. Wang, J.; Tao, Q.; Wang, H.; Wang, Z.; Wu, F.; Pan, Y.; Tao, L.; Xiong, S.; Wang, Y.; Zhai, Z. Elevated IL-35 in bone marrow of the patients with acute myeloid leukemia. Hum. Immunol. 2015, 76, 681-686. [CrossRef]

18. Tao, Q.; Pan, Y.; Wang, Y.; Wang, H.; Xiong, S.; Lili, T.; Wang, J.; Tao, L.; Wang, Z.; Wu, F.; et al. Regulatory T cells-derived IL-35 promotes the growth of adult acute myeloid leukemia blasts. Int. J. Cancer 2015, 137, 2384-2393. [CrossRef]

19. Wang, Z.; Liu, J.-Q.; Liu, Z.; Shen, R.; Zhang, G.; Xu, J.; Basu, S.; Feng, Y.; Bai, X.-F. Tumor-Derived IL-35 Promotes Tumor Growth by Enhancing Myeloid Cell Accumulation and Angiogenesis. J. Immunol. 2013, 190, 2415-2423. [CrossRef]

20. Campo, E.; Swerdlow, S.H.; Harris, N.L.; Pileri, S.; Stein, H.; Jaffe, E.S. The 2008 WHO classification of lymphoid neoplasms and beyond: Evolving concepts and practical applications. Blood 2011, 117, 5019-5032. [CrossRef]

21. Younes, A.; Hilden, P.; Coiffier, B.; Hagenbeek, A.; Salles, G.; Wilson, W.; Seymour, J.F.; Kelly, K.; Gribben, J.; Pfreunschuh, M.; et al. International Working Group consensus response evaluation criteria in lymphoma (RECIL 2017). Ann. Oncol. 2017, 28, 1436-1447. [CrossRef]

22. Bayne, L.J.; Beatty, G.L.; Jhala, N.; Clark, C.E.; Rhim, A.D.; Stanger, B.Z.; Vonderheide, R.H. Tumor-Derived GranulocyteMacrophage Colony-Stimulating Factor Regulates Myeloid Inflammation and T Cell Immunity in Pancreatic Cancer. Cancer Cell 2012, 21, 822-835. [CrossRef]

23. Kanai, K.; Park, A.-M.; Yoshida, H.; Tsunoda, I.; Yoshie, O. IL-35 Suppresses Lipopolysaccharide-Induced Airway Eosinophilia in EBI3-Deficient Mice. J. Immunol. 2016, 198, 119-127. [CrossRef]

24. Shu, C.-C.; Pan, S.-W.; Feng, J.-Y.; Wang, J.-Y.; Chan, Y.-J.; Yu, C.-J.; Su, W.-J. The Clinical Significance of Programmed Death-1, Regulatory T Cells and Myeloid Derived Suppressor Cells in Patients with Nontuberculous Mycobacteria-Lung Disease. J. Clin. Med. 2019, 8, 736. [CrossRef]

25. Malek, E.; de Lima, M.; Letterio, J.J.; Kim, B.-G.; Finke, J.H.; Driscoll, J.J.; Giralt, S.A. Myeloid-derived suppressor cells: The green light for myeloma immune escape. Blood Rev. 2016, 30, 341-348. [CrossRef] 
26. Wu, P.; Wu, D.; Ni, C.; Ye, J.; Chen, W.; Hu, G.; Wang, Z.; Wang, C.; Zhang, Z.; Xia, W.; et al. GammadeltaT17 cells promote the accumulation and expression of myeloid-derived suppressor cells in human colorectal cancer. Immunity 2014, 40, 785-800. [CrossRef]

27. Gonda, K.; Shibata, M.; Ohtake, T.; Matsumoto, Y.; Tachibana, K.; Abe, N.; Ohto, H.; Sakurai, K.; Takenoshita, S. Myeloid-derived suppressor cells are increased and correlated with type 2 immune responses, malnutrition, inflammation, and poor prognosis in patients with breast cancer. Oncol. Lett. 2017, 14, 1766-1774. [CrossRef]

28. Haverkamp, J.M.; Smith, A.M.; Weinlich, R.; Dillon, C.P.; Qualls, J.E.; Neale, G.; Koss, B.; Kim, Y.; Bronte, V.; Herold, M.J.; et al. Myeloid-Derived Suppressor Activity Is Mediated by Monocytic Lineages Maintained by Continuous Inhibition of Extrinsic and Intrinsic Death Pathways. Immunity 2014, 41, 947-959. [CrossRef] [PubMed]

29. Azzaoui, I.; Uhel, F.; Rossille, D.; Pangault, C.; Dulong, J.; Le Priol, J.; Lamy, T.; Houot, R.; Le Gouill, S.; Cartron, G.; et al. T-cell defect in diffuse large B-cell lymphomas involves expression of myeloid-derived suppressor cells. Blood 2016, 128, 1081-1092. [CrossRef] [PubMed]

30. Wu, C.; Wu, X.; Liu, X.; Yang, P.; Xu, J.; Chai, Y.; Guo, Q.; Wang, Z.; Zhang, L. Prognostic Significance of Monocytes and Monocytic Myeloid-Derived Suppressor Cells in Diffuse Large B-Cell Lymphoma Treated with R-CHOP. Cell. Physiol. Biochem. 2016, 39, 521-530. [CrossRef] [PubMed]

31. Wu, C.; Wu, X.; Zhang, X.; Chai, Y.; Guo, Q.; Li, L.; Yue, L.; Bai, J.; Wang, Z.; Zhang, L. Prognostic significance of peripheral monocytic myeloid-derived suppressor cells and monocytes in patients newly diagnosed with diffuse large b-cell lymphoma. Int. J. Clin. Exp. Med. 2015, 8, 15173-15181.

32. Wang, K.; Gong, H.; Chai, R.; Yuan, H.; Chen, Y.; Liu, J. RETRACTED: Aberrant frequency of IL-35 producing B cells in colorectal cancer patients. Cytokine 2018, 102, 206-210. [CrossRef]

33. Long, J.; Guo, H.; Cui, S.; Zhang, H.; Liu, X.; Li, D.; Han, Z.; Xi, L.; Kou, W.; Xu, J.; et al. IL-35 expression in hepatocellular carcinoma cells is associated with tumor progression. Oncotarget 2016, 7, 45678-45686. [CrossRef]

34. Bronte, V.; Brandau, S.; Chen, S.-H.; Colombo, M.P.; Frey, A.B.; Greten, T.F.; Mandruzzato, S.; Murray, P.J.; Ochoa, A.; OstrandRosenberg, S.; et al. Recommendations for myeloid-derived suppressor cell nomenclature and characterization standards. Nat. Commun. 2016, 7, 12150. [CrossRef] 